\title{
Pythagorean Isoparametric Hypersurfaces in Riemannian and Lorentzian Space Forms
}

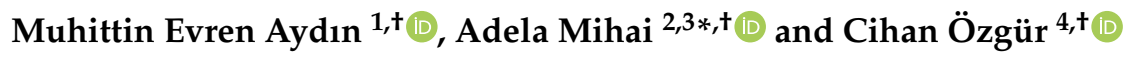 \\ 1 Department of Mathematics, Firat University, Elazig 23119, Turkey; meaydin@firat.edu.tr \\ 2 Department of Mathematics and Computer Science, Technical University of Civil Engineering Bucharest, \\ 020396 Bucharest, Romania \\ 3 Interdisciplinary Doctoral School, Transilvania University of Braşov, 500036 Braşov, Romania \\ 4 Department of Mathematics, İzmir Democracy University, İzmir 35140, Turkey; cihan.ozgur@idu.edu.tr \\ * Correspondence: adela.mihai@utcb.ro or adela.mihai@unitbv.ro \\ + These authors contributed equally to this work.
}

check for updates

Citation: Aydın, M.E.; Mihai, A.; Özgür, C. Pythagorean Isoparametric Hypersurfaces in Riemannian and Lorentzian Space Forms. Axioms 2022, 11, 59. https://doi.org/10.3390/ axioms11020059

Academic Editor: Mica Stankovic

Received: 1 January 2022

Accepted: 27 January 2022

Published: 30 January 2022

Publisher's Note: MDPI stays neutral with regard to jurisdictional claims in published maps and institutional affiliations.

Copyright: (c) 2022 by the authors. Licensee MDPI, Basel, Switzerland. This article is an open access article distributed under the terms and conditions of the Creative Commons Attribution (CC BY) license (https:// creativecommons.org/licenses/by/ $4.0 /)$.

\begin{abstract}
We introduce the notion of a Pythagorean hypersurface immersed into an $(n+1)$-dimensional pseudo-Riemannian space form of constant sectional curvature $c \in\{-1,0,1\}$. By using this definition, we prove in Riemannian setting that if an isoparametric hypersurface is Pythagorean, then it is totally umbilical with sectional curvature $\varphi+c$, where $\varphi$ is the Golden Ratio. We also extend this result to Lorentzian ambient space, observing the existence of a non totally umbilical model.
\end{abstract}

Keywords: Pythagorean triple; Golden Ratio; isoparametric hypersurface; shape operator; GaussKronecker curvature

\section{Introduction}

Let $a, b, c$ be positive integers. The triple $\{a, b, c\}$ is said to be Pythagorean, if $a^{2}+b^{2}=c^{2}$. If $\{a, b, c\}$ is Pythagorean, then so is $\{k a, k b, k c\}$ for any positive integer $k$. If $a, b, c$ have no common factor, then $\{a, b, c\}$ is said to be primitive Pythagorean, for example $\{3,4,5\}$, $\{5,12,13\},\{7,24,25\}$, etc. Let $m, n$ be positive integers such that $m>n, m+n \equiv 1(\bmod 2)$ and $m, n$ mutually prime. Then the triple $\left\{m^{2}-n^{2}, 2 m n, m^{2}+n^{2}\right\}$ is a primitive Pythagorean triple. In the previous examples, $(m, n)$ are respectively $(2,1),(3,2)$ and $(4,3)$, see [1]. The reference [2] states that the method of finding the primitive Pythagorean triples is attributed to the Indian mathematician Brahmagupta (598-665 AD).

Nice relations between the Pythagorean triples and square matrices appear in the literature. We first recall the notion of Pythagorean triple preserving matrix ([2-4]): let $\{a, b, c\}$ be some primitive Pythagorean triple and $\mathbf{X}=\left[\begin{array}{lll}a & b & c\end{array}\right]$. Then a square matrix $\mathbf{A}$ of order 3 is called Pythagorean triple preserving matrix, if $\mathbf{X} \cdot \mathbf{A}$ is a Pythagorean triple for each $\mathbf{X}$, where $\cdot$ is the matrix multiplication. For example, let

$$
\mathbf{A}=\left[\begin{array}{ccc}
1 & 2 & 2 \\
-2 & -1 & -2 \\
2 & 2 & 3
\end{array}\right]
$$

Then $\left[\begin{array}{lll}3 & 4 & 5\end{array}\right] \mathbf{A}=\left[\begin{array}{lll}5 & 12 & 13\end{array}\right]$ and $\left[\begin{array}{lll}5 & 12 & 13\end{array}\right] \mathbf{A}=\left[\begin{array}{lll}7 & 24 & 25\end{array}\right]$, etc.

We next recall the generalization of Pythagorean triples to the triple of square matrices of order $n$ with integer entries: let $\mathbf{A}, \mathbf{B}, \mathbf{C}$ be square matrices of order $n$ with integer entries. Then $\{\mathbf{A}, \mathbf{B}, \mathbf{C}\}$ is called a matrix Pythagorean triple [5], if $\mathbf{A}^{2}+\mathbf{B}^{2}=\mathbf{C}^{2}$, where $\mathbf{A}^{2}=\mathbf{A} \cdot \mathbf{A}$, etc. A trivial example in the case $n=2$ is the following: let $\{a, b, c\}$ be a Pythagorean triple. Then $\mathbf{A}=\operatorname{diag}\left[\begin{array}{ll}a & 0\end{array}\right], \mathbf{B}=\operatorname{diag}\left[\begin{array}{ll}b & 0\end{array}\right], \mathbf{C}=\operatorname{diag}\left[\begin{array}{ll}c & 0\end{array}\right]$ is a matrix Pythagorean triple. The details of construction of non-trivial examples may be found in [5].

Due to the Pythagorean theorem [1], the Pythagorean triples have a brilliant geometrical meaning, i.e., the lengths of the sides of a right triangle, when they are integers, are a 
Pythagorean triple. Nevertheless, the origin of matrix Pythagorean triples is not based on any geometrical meaning.

Most recently, in [6], the first and second authors gave a geometrical meaning to the matrix Pythagorean triples by using the differential geometry of surfaces.

More explicitly, let $\mathbb{M}^{3}(c)$ be a 3-dimensional Riemannian space form of constant sectional curvature $c \in\{-1,0,1\}$ and $M^{2}$ an isometrically immersed surface into $\mathbb{M}^{3}(c)$. Then $M^{2}$ basically has three symmetric bilinear forms and corresponding square matrices, justifying the following definition: a surface $M^{2}$ immersed into $\mathbb{M}^{3}(c)$ satisfying the socalled Pythagorean-like formula

$$
\mathbf{I}^{2}+\mathbf{I I}^{2}=\mathbf{I I I}^{2}
$$

was considered in [6], where $\mathbf{I}^{2}, \mathbf{I I}^{2}$ and $\mathbf{I I I} \mathbf{I}^{2}$ are the squares of the matrices corresponding to the first, second and third fundamental forms of $M^{2}$, respectively. We point out that $\{\mathbf{I}, \mathbf{I I}, \mathbf{I I I}\}$ is a matrix Pythagorean triple and hereinafter we briefly call such a surface Pythagorean. The entries in the original definition of matrix Pythagorean triples are integer, while those, in our case, are allowed to be real numbers.

An example of Pythagorean surface is the following: let $c=0$ and $\mathbb{S}^{2}(r)$ be the sphere of radius $r$ centered at origin. It is immediate that $\mathbf{I I}=(1 / r) \mathbf{I}$ and $\mathbf{I I I}=\left(1 / r^{2}\right) \mathbf{I}$ ([7]). Then $\mathbb{S}^{2}(r)$ is Pythagorean if and only if the algebraic equation of degree 4 holds

$$
r^{4}+r^{2}-1=0,
$$

which only allows the positive real root $r=[(-1+\sqrt{5}) / 2]^{1 / 2}$.

Denote by $\varphi:=(1+\sqrt{5}) / 2$ the Golden Ratio and by $\Phi:=(-1+\sqrt{5}) / 2$ the conjugate Golden Ratio. Hence the Gaussian curvature of this Pythagorean sphere $\mathbb{S}^{2}\left(\Phi^{1 / 2}\right)$ is $G=\varphi$.

Let $\mathbb{M}^{3}(c)$ be one of the standard complete simply-connected models, i.e. the hyperbolic space $\mathbb{H}^{3}(-1)$, Euclidean space $\mathbb{R}^{3}$ and 3-sphere $\mathbb{S}^{3}(1)$. In [6], the authors classified compact Pythagorean surfaces immersed into $\mathbb{M}^{3}(c)$, obtaining that a compact Pythagorean surface is a totally umbilical round sphere with Gaussian curvature $G=\varphi+c$.

Since the early ages, scientists, philosophers, artists have shown interest in the Pythagorean Theorem and Golden Ratio $\varphi=(1+\sqrt{5}) / 2=1.61803398874989 \ldots$ ([8]). The importance of these remarkable concepts in mathematics is keenly expressed by Johannes Kepler (1571-1630) (see [9]):

"Geometry has two great treasures; one is the Theorem of Pythagoras; the other, the division of a line into extreme and mean ratio. The first we may compare to a measure of gold; the second we may name a precious jewel".

In this paper, we generalize to higher dimensions the Pythagorean-like formula. We notice that, in terms of the corresponding matrix $\mathbf{A}$ to shape operator, the Pythagorean-like formula is equivalent with:

$$
\mathbf{I}^{2}+(\mathbf{I} \cdot \mathbf{A})^{2}=\left(\mathbf{I} \cdot \mathbf{A}^{2}\right)^{2} .
$$

We use relation (1) for generalization and give the following

Definition 1. Let $M$ be an orientable non-degenerate hypersurface immersed into an $(n+1)$ dimensional pseudo-Riemannian space form $\mathbb{M}^{n+1}(c)$ of constant sectional curvature $c \in\{-1,0,1\}$. Denote by $\mathbf{I}$ and $\mathbf{A}$ the corresponding matrices to the induced pseudo-Riemannian metric and shape operator of $M$, respectively. Then $M$ is called a Pythagorean hypersurface if (1) holds.

We point out that the orientability assures the global properties of our notions.

The above example of Pythagorean sphere can be extended to higher dimensions as follows: 
Example 1. Let $c=0$ and $\mathbb{S}^{n}(r)$ be the hypersphere in $\mathbb{R}^{n+1}$ of radius $r$ centered at the origin. Then $\mathbf{A}=(1 / r) \mathcal{I}_{n}$, for the unit matrix $\mathcal{I}_{n}$ of order $n$ [10]. $\mathbb{S}^{n}(r)$ is Pythagorean if and only if $r=\Phi^{1 / 2}$, or, equivalently, $K=\varphi$, where $K$ is the sectional curvature. The scalar curvature of this Pythagorean hypersphere is $\tau=\left(\begin{array}{l}n \\ 2\end{array}\right) \varphi$ and the Gauss-Kronecker curvature is $G=\varphi^{n / 2}$. In such a case, $G$ may be expressed in terms of the Fibonacci numbers by:

$$
G=\left(F_{n-1}+F_{n} \varphi\right)^{1 / 2},
$$

where $F_{n-1}$ and $F_{n}$ are the elements of the Fibonacci sequence [8]. Particularly, for $n=2$, we have $G=\varphi$.

As can be seen in Example 1, the totally umbilical hypersurface $\mathbb{S}^{n}\left(\Phi^{1 / 2}\right)$ is Pythagorean. Besides this feature, $\mathbb{S}^{n}\left(\Phi^{1 / 2}\right)$ is an isoparametric hypersurface in Riemannian setting, namely a hypersurface having constant principal curvatures [11].

Starting from this point of view, we will consider Pythagorean isoparametric hypersurfaces in a Riemannian space form $\mathbb{M}^{n+1}(c)$ and prove that such a hypersurface is totally umbilical with sectional curvature $K=\varphi+c$. Remark that in case $c=1$, we will allow the hypersurface to have at most two distinct principal curvatures. Furthermore, we will extend the result to Lorentzian ambient space observing, in contrast to Riemannian setting, the existence of a Pythagorean hypersurface which is non totally umbilical.

It is worth to point out that the Pythagorean-like formula is similar to the so-called metallic shaped hypersurface equation: $([12,13])$

$$
a \mathcal{I}_{n}+b \mathbf{A}=\mathbf{A}^{2},
$$

where $a, b$ are some positive integers. A hypersurface with (2) is said to be metallic shaped. In the particular case $a=b=1$ it is called a golden shaped hypersurface [14]. In the cited papers, the authors obtained that the metallic and golden shaped hypersurfaces are isoparametric and they provided full classifications.

\section{Preliminaries}

Let $(\tilde{M}, \tilde{g})$ be an $(n+1)$-dimensional (pseudo) Riemannian manifold, where $\tilde{g}$ is a symmetric non-degenerate $(0,2)$ tensor field of constant index. In particular if the index is 0 (resp. 1), then $\tilde{M}$ is said to be Riemannian (resp. Lorentzian) manifold. Let $\tilde{\nabla}$ be Levi-Civita connection of $\tilde{M}$. The Riemannian curvature tensor of type (3) is defined by:

$$
\tilde{R}(X, Y) Z=\tilde{\nabla}_{X} \tilde{\nabla}_{Y} Z-\tilde{\nabla}_{Y} \tilde{\nabla}_{X} Z-\tilde{\nabla}_{[X, Y]} Z,
$$

where $X, Y, Z$ are arbitrary tangent vector fields to $\tilde{M}$ and [,] bracket operation. Let $T_{p} \tilde{M}$ be tangent space of $\tilde{M}$ at $p \in \tilde{M}$ and $\Gamma$ a non-degenerate plane section of $T_{p} \tilde{M}$ with given a basis $\{u, v\}$, namely:

$$
\tilde{g}(u, u) \tilde{g}(v, v)-\tilde{g}(u, v)^{2} \neq 0 .
$$

The sectional curvature $K(\Gamma)$ of $\Gamma$ is:

$$
K(u, v)=\frac{\tilde{g}(\tilde{R}(u, v) v, u)}{\tilde{g}(u, u) \tilde{g}(v, v)-\tilde{g}(u, v)^{2}} .
$$

Let $\left\{e_{1}, \ldots, e_{n+1}\right\}$ be an orthonormal frame of $\tilde{M}$. Then the scalar curvature is:

$$
\tau=\sum_{i<j} K\left(e_{i}, e_{j}\right)
$$

where $i, j \in\{1, \ldots, n+1\}$. 
Let $M$ be an orientable non-degenerate hypersurface immersed into $\tilde{M}$ and $\xi$ a unit normal vector field over $M$. Denote by $X, Y$ and $\nabla$ arbitrary two tangent vector fields to $M$ and the induced Levi-Civita connection. Then the formula of Gauss is

$$
\tilde{\nabla}_{X} Y=\nabla_{X} Y+h(X, Y),
$$

where $h$ is the second fundamental form of $M$ [15].

Notice that $\tilde{g}(h(X, Y), \tilde{\xi})=g(A X, Y)$, where $A$ is a $(1,1)$ tensor field, called shape operator or Weingarten map and $g$ is the induced pseudo-Riemannian metric tensor. The eigenvalues $\kappa_{1}, \ldots, \kappa_{n}$ of $A$ are called the principal curvatures of $M . M^{n}$ is said to be totally geodesic if $A$ vanishes identically and totally umbilical if $A=\lambda \mathcal{I}$, where $\mathcal{I}$ is the identity on the tangent bundle of $M$ and $\lambda$ a real constant [15]. Furthermore, the $p$-fundamental form of $M$ is introduced by $\chi^{p}(X, Y)=g\left(A^{p-1} X, Y\right)$, where $A^{p-1}=\underbrace{A \circ A \circ \ldots \circ A}_{p-1 \text { times }}[16]$.

Let $R$ be the Riemannian curvature tensor of $M$ and $X, Y, Z, W$ be arbitrary tangent vector fields to $M$. Then the equation of Gauss is:

$$
g(R(X, Y) Z, W)=\tilde{g}(\tilde{R}(X, Y) Z, W)+\tilde{g}(h(X, W), h(Y, Z))-\tilde{g}(h(X, Z), h(Y, W)) .
$$

Denote by $\mathcal{I}_{n}$ and $\mathbf{0}_{n}$ the unit and zero matrices of order $n$, respectively. We next provide the following remark, which we will use later:

Remark 1. Let $M$ be an orientable non-degenerate hypersurface immersed into $\tilde{M}$. Denote by $\mathbf{I}$ and $\mathbf{A}$ the corresponding matrices to the induced metric tensor and shape operator. Then the Pythagorean-like formula (1) does not hold for $\mathbf{A}=\mathbf{0}_{n}$ and $\mathbf{A}= \pm \mathcal{I}_{n}$.

The proof is by contradiction. If $\mathbf{A}=\mathbf{0}_{n}$, then from (1), it follows that $\mathbf{I}^{2}=\mathbf{0}_{n}$, which implies $\operatorname{det} \mathbf{I}=0$. This is a contradiction because $M$ is non-degenerate, namely $\mathbf{I}$ is invertible. The same contradiction may be easily obtained by assuming $\mathbf{A}= \pm \mathcal{I}_{n}$.

We end the section by highlighting that the original definition of Pythagorean-like formula (see [6]) uses the basis of tangent vectors to the coordinate curves of given surface and in such a case the matrix $\mathbf{A}$ is not necessary to be diagonal. In order to better adapt to the study of isoparametric hypersurfaces in higher dimensions, we will get the basis of principal directions to use (1).

\section{Riemannian Settings}

Let $\mathbb{M}^{n+1}(c)$ denote an $(n+1)$-dimensional Riemannian space form with $c \in\{-1,0,1\}$. The standard models are the hyperbolic space $\mathbb{H}^{n+1}(-1)$, Euclidean space $\mathbb{R}^{n+1}$ and $(n+1)$-sphere $\mathbb{S}^{n+1}(1)$. Throughout this section we will be interested in these standard models.

In the upper half space model, the hyperbolic space is:

$$
\mathbb{H}^{n+1}(-1)=\left\{\mathbf{u}=\left(u_{1}, \ldots, u_{n+2}\right) \in \mathbb{R}^{n+2}: \sum_{i=1}^{n+1} u_{i}^{2}-u_{n+2}^{2}=-1, u_{n+2}>0\right\} .
$$

The $(n+1)$-sphere $\mathbb{S}^{n+1}$ is the unit hypersphere of $\mathbb{R}^{n+2}$, namely:

$$
\mathbb{S}^{n+1}(1)=\left\{\mathbf{u} \in \mathbb{R}^{n+2}: \sum_{i=1}^{n+2} u_{i}^{2}=1\right\}
$$

Recall that a hypersurface $M$ immersed into $\mathbb{M}^{n+1}(c)$ having constant principal curvatures is said to be isoparametric [11]. In the following, we investigate Pythagorean isoparametric hypersurfaces in three separate subsections. 


\subsection{Hypersurfaces of the Euclidean Space}

Let $M$ be an isoparametric hypersurface in $\mathbb{R}^{n+1}$ and $\mathbf{A}$ the corresponding matrix of its shape operator. Hence, $M$ is either a hyperplane with $\mathbf{A}=\mathbf{0}_{n}$ or the hypersphere $\mathbb{S}^{n}(r)$ with $\mathbf{A}=\frac{1}{r} \mathcal{I}_{n}$ or a spherical cylinder $\mathbb{S}^{k}(r) \times \mathbb{R}^{n-k}$ with $\mathbf{A}=\frac{1}{r} \mathcal{I}_{k} \oplus \mathbf{0}_{n-k}$ ([11]). Here $\oplus$ denotes the direct sum of matrices. Therefore we have the next result:

Theorem 1. Let $M$ be a complete isoparametric hypersurface of $\mathbb{R}^{n+1}$. If $M$ is Pythagorean, then it is isometric to $\mathbb{S}^{n}(\sqrt{\Phi})$.

Proof. Assume that $M$ is Pythagorean. According to Remark 1, we may neglect the case that $M$ is a hyperplane. Suppose that $M$ is a spherical cylinder. Then we have $\mathbf{A}^{2}=\left(1 / r^{2}\right) \mathcal{I}_{k} \oplus \mathbf{0}_{n-k}$ and

$$
\mathbf{I} \cdot \mathbf{A}=\frac{1}{r} \mathbf{I}_{k} \oplus \mathbf{0}_{n-k}
$$

where $\mathbf{I}_{k}$ is the block matrix consisting of the first $k$ rows and columns of $\mathbf{I}$. Then (1) gives:

$$
\mathbf{I}^{2}=-\left(\frac{1}{r^{2}}-\frac{1}{r^{4}}\right) \mathbf{I}_{k}^{2} \oplus \mathbf{0}_{n-k} .
$$

Because the induced metric is Riemannian, the matrix $\mathbf{I}$ is invertible and taking determinant of both sides of this equation we get $\operatorname{det} \mathbf{I}=0$, which is not possible. Then $M$ cannot be a spherical cylinder. The last scenario for $M$ is being $\mathbb{S}^{n}(r)$ with $\mathbf{A}=\frac{1}{r} \mathcal{I}_{n}$. In such a case, (1) yields:

$$
\left(r^{4}+r^{2}-1\right) \mathbf{I}^{2}=\mathbf{0}_{n} .
$$

Since I is invertible, we have the algebraic equation of degree 4

$$
r^{4}+r^{2}-1=0,
$$

which has the only positive real root $r=\sqrt{\Phi}$. This completes the proof.

As emphasized in Example 1, the sectional curvature of the Pythagorean hypersphere $\mathbb{S}^{n}(\sqrt{\Phi})$ is $K=\varphi$ and the scalar curvature is $\tau=\left(\begin{array}{l}n \\ 2\end{array}\right) \varphi$. Furthermore, the Gauss-Kronecker curvature which corresponds to the determinant of $\mathbf{A}$ (in the Euclidean setting) is $G=\varphi^{n / 2}$.

\subsection{Hypersurfaces of the Hyperbolic Space}

Let $M$ be an isoparametric hypersurface in $\mathbb{H}^{n+1}(-1):=\mathbb{H}^{n+1}$ and $\mathbf{u}=\left(u_{1}, \ldots, u_{n+2}\right) \in$ $\mathbb{R}^{n+2}$. Then one of the following situations occurs ([17]):

1. $M=\left\{\mathbf{u} \in \mathbb{H}^{n+1} \subset \mathbb{R}^{n+2} \mid u_{k}=0,1 \leq k \leq n+1\right\}$ with $\mathbf{A}=\mathbf{0}_{k}$;

2. $M=\left\{\mathbf{u} \in \mathbb{H}^{n+1} \subset \mathbb{R}^{n+2} \mid u_{k}=r, r \in(1, \infty), 1 \leq k \leq n+1\right\}$ with $\mathbf{A}=\frac{\sqrt{r^{2}-1}}{r} \mathcal{I}_{n} ;$

3. $M=\left\{\mathbf{u} \in \mathbb{H}^{n+1} \subset \mathbb{R}^{n+2} \mid u_{n+2}=u_{n+1}+1\right\}$ with $\mathbf{A}=\mathcal{I}_{n}$;

4. $M=\mathbb{H}^{n+1} \cap \mathbb{S}^{n}(r)=\left\{\mathbf{u} \in \mathbb{H}^{n+1} \subset \mathbb{R}^{n+2} \mid u_{1}^{2}+\ldots+u_{n+1}^{2}=r^{2}, r>0\right\}$ with

$\mathbf{A}=\frac{\sqrt{r^{2}+1}}{r} \mathcal{I}_{n}$;

5. $\quad M=\mathbb{H}^{n+1} \cap \mathbb{S}^{k}(r)=\left\{\mathbf{u} \in \mathbb{H}^{n+1} \subset \mathbb{R}^{n+2} \mid u_{1}^{2}+\ldots+u_{k+1}^{2}=r^{2}, r>0\right\}$ with $\mathbf{A}=\frac{\sqrt{r^{2}+1}}{r} \mathcal{I}_{k} \oplus \frac{r}{\sqrt{r^{2}+1}} \mathcal{I}_{n-k}$

Notice that the above hypersurfaces in items (2), (3), (4) are isometric to $\mathbb{H}^{n}\left(-\frac{1}{r^{2}}\right)$, $\mathbb{R}^{n}$ and $\mathbb{S}^{n}(r)$, respectively. In the following we classify the Pythagorean isoparametric hypersurfaces in $\mathbb{H}^{n+1}$ :

Theorem 2. Let $M$ be a complete isoparametric hypersurface of $\mathbb{H}^{n+1}$. If $M$ is Pythagorean, then it is isometric to $\mathbb{S}^{n}(\sqrt{\varphi})$. 
Proof. By the hypothesis, $M$ may be one of the above items (1)-(5). However, according to Remark 1, it is sufficient to consider the items (2), (4) and (5) and the corresponding cases:

Case (2). $\mathbf{A}=\frac{\sqrt{r^{2}-1}}{r} \mathcal{I}_{n}$, with $r \in(1, \infty)$. Then (1) implies:

$$
\left(r^{4}+r^{2}-1\right) \mathbf{I}^{2}=\mathbf{0}_{n} .
$$

By using the positive definiteness of the induced metric tensor, we deduce:

$$
r^{4}+r^{2}-1=0,
$$

which gives a contradiction because the equation has no roots in $(1, \infty)$.

Case (4). $\mathbf{A}=\frac{\sqrt{r^{2}+1}}{r} \mathcal{I}_{n}, r>0$. Then (1) is:

$$
\left(r^{4}-r^{2}-1\right) \mathbf{I}^{2}=\mathbf{0}_{n}
$$

which gives $r^{4}-r^{2}-1=0$. This algebraic equation has the only positive real $\operatorname{root} r=\sqrt{\varphi}$, which proves the result.

Case (5). $\mathbf{A}=\frac{\sqrt{r^{2}+1}}{r} \mathcal{I}_{k} \oplus \frac{r}{\sqrt{r^{2}+1}} \mathcal{I}_{n-k}, r>0$. We may choose an orthogonal frame of $M$ such that $\mathbf{I}$ is diagonal and $\mathbf{A}$ has the form of our assumption. By using the property that a diagonal matrix commutes with other diagonal matrices, we conclude from (1) that:

$$
\mathbf{I}^{2}\left(-\mathcal{I}_{n}-\mathbf{A}^{2}+\mathbf{A}^{4}\right)=\mathbf{0}_{n}
$$

implying

$$
\begin{array}{r}
r^{4}+3 r^{2}+1=0 \\
r^{4}-r^{2}-1=0 .
\end{array}
$$

From the previous system of two equations, one gets the contradiction $4 r^{2}+2=0$. This implies that the system has no real root.

Notice that one writes $\mathbf{A}=\sqrt{\varphi} \mathcal{I}_{n}$ for the Pythagorean hypersurface $\mathbb{S}^{n}(\sqrt{\varphi})$ in $\mathbb{H}^{n+1}$ and hence its sectional curvature $K=\varphi-1$ and the scalar curvature is $\tau=\left(\begin{array}{c}n \\ 2\end{array}\right)(\varphi-1)$.

\subsection{Hypersurfaces of the Sphere}

Let $M$ be an isoparametric hypersurface of $\mathbb{S}^{n+1}(1):=\mathbb{S}^{n+1}$ having at most two distinct principal curvatures. Then it is given by one of the following forms (see $[17,18])$

1. $M=\left\{\mathbf{u} \in \mathbb{S}^{n+1} \subset \mathbb{R}^{n+2} \mid u_{n+2}=\sqrt{1-r^{2}}, r \in(0,1)\right\}$ with $\mathbf{A}=\frac{\sqrt{1-r^{2}}}{r} \mathcal{I}_{n} ;$

2. $M=\mathbb{S}^{k}\left(r_{1}\right) \times \mathbb{S}^{n-k}\left(r_{2}\right)$ with $r_{1}^{2}+r_{2}^{2}=1$ and $1 \leq k<n$.

In (1) $M$ is isometric to $\mathbb{S}^{n}$; in (2) $M$ is the generalized Clifford torus, with $r_{1}=\frac{1}{\sqrt{1+\lambda_{1}^{2}}}$ and $r_{2}=\frac{1}{\sqrt{1+\lambda_{2}^{2}}}$, such that $\lambda_{1}=r_{2} / r_{1}$ and $\lambda_{2}=-r_{1} / r_{2}$ are the principal curvatures and $\lambda_{1} \lambda_{2}=-1$.

We present the Pythagorean isoparametric hypersurfaces of $\mathbb{S}^{n+1}$ as follows:

Theorem 3. Let $M$ be a complete isoparametric hypersurface with at most two distinct principal curvatures in $\mathbb{S}^{n+1}$. If $M$ is Pythagorean, then it is isometric to $S^{n}(\Phi)$.

Proof. By the assumption, we have the above items (1) and (2). If $\mathbf{A}=\frac{\sqrt{1-r^{2}}}{r} \mathcal{I}_{n}, r \in(0,1)$, then (1) implies:

$$
\left(r^{4}-3 r^{2}+1\right) \mathbf{I}^{2}=\mathbf{0}_{n}
$$


from where $r^{4}-3 r^{2}+1=0$, because $\mathbf{I}$ is invertible. The only permissible root is $r=\Phi$, proving the result.

For the item (2), as in the proof of Theorem 2, an orthogonal frame of $M$ may be choosed such that $\mathbf{I}$ is diagonal and $\mathbf{A}$ has the form of our assumption. Then (1) is:

$$
\mathbf{I}^{2}\left(-\mathcal{I}-\mathbf{A}^{2}+\mathbf{A}^{4}\right)=\mathbf{0}_{n}
$$

which gives

$$
\begin{aligned}
& r_{1}^{4}+r_{1}^{2} r_{2}^{2}=r_{2}^{4} \\
& r_{2}^{4}+r_{1}^{2} r_{2}^{2}=r_{1}^{4} .
\end{aligned}
$$

By these equations, the contradiction $2 r_{1}^{2} r_{2}^{2}=0$ is obtained.

Since the shape operator matrix of the Pythagorean hypersurface $\mathbb{S}^{n}(\Phi)$ in $\mathbb{S}^{n+1}$ is $\mathbf{A}=$ $(1 / \sqrt{\Phi}) \mathcal{I}_{n}$, the sectional curvature is $K=\varphi+1$ and the scalar curvature $\tau=\left(\begin{array}{l}n \\ 2\end{array}\right)(\varphi+1)$.

Summarizing Theorems 1-3, we have the following classification result:

Theorem 4 (Classification of Pythagorean isoparametric hypersurfaces of a Riemannian space form). Let $\mathbb{M}^{n+1}(c), c \in\{-1,0,1\}$, be an $(n+1)$-dimensional Riemannian space form and $M$ a complete isoparametric hypersurface of $\mathbb{M}^{n+1}(c)$ (if the ambient space is the $(n+1)$ sphere, one considers $M$ having at most two distinct principal curvatures). Then $M$ is Pythagorean if and only if it is totally umbilical with $K=\varphi+c$.

\section{Lorentzian Settings}

Let $\mathbb{M}_{1}^{n+1}(c), c \in\{-1,0,1\}$, be an $(n+1)$-dimensional Lorentzian space form. As in the Riemannian setting, we will be interested in the standard models, namely the Minkowski space $\mathbb{R}_{1}^{n+1}$, anti-de Sitter space

$$
\mathbb{H}_{1}^{n+1}(-1)=\left\{\mathbf{u}=\left(u_{1}, \ldots, u_{n+2}\right) \in \mathbb{R}_{1}^{n+2}:-u_{1}^{2}+\sum_{i=2}^{n+2} u_{i}^{2}=-1\right\}
$$

and de Sitter space

$$
\mathbb{S}_{1}^{n+1}(1)=\left\{\mathbf{u} \in \mathbb{R}_{1}^{n+2}:-u_{1}^{2}+\sum_{i=2}^{n+2} u_{i}^{2}=1\right\}
$$

Here the non-degenerate metric of $\mathbb{R}_{1}^{n+1}$ is of the form $-d u_{1}^{2}+\sum_{i=2}^{n+2} d u_{i}^{2}$.

Let $M$ be a hypersurface immersed into $\mathbb{M}_{1}^{n+1}(c)$. In the case where the shape operator $\mathbf{A}$ is diagonalizable, the hypersurface is said to be isoparametric if $\mathbf{A}$ has constant principal curvatures [19]. In the present paper, we consider isoparametric hypersurfaces with at most two mutually distinct constant principal curvatures in $\mathbb{M}_{1}^{n+1}(c)$. Similar with the Riemannian setting, we will investigate Pythagorean isoparametric hypersurfaces of $\mathbb{M}_{1}^{n+1}(c)$.

\subsection{Hypersurfaces of the Minkowski Space}

An isoparametric hypersurface $M$ with at most two mutually distinct principal curvatures in $\mathbb{R}_{1}^{n+1}$ is given by one of the following forms ([20]):

(1) $\mathbb{R}^{n}=\left\{\mathbf{u} \in \mathbb{R}_{1}^{n+1} \mid u_{1}=0\right\}$ with $\mathbf{A}=\mathbf{0}_{n}$,

(2) $\mathbb{H}^{n}(r)=\left\{\mathbf{u} \in \mathbb{R}_{1}^{n+1} \mid-u_{1}^{2}+\sum_{i=2}^{n+1} u_{i}^{2}=\frac{1}{r}, r<0\right\}$ with $\mathbf{A}= \pm \sqrt{-r} \mathcal{I}_{n}$,

(3) $\mathbb{R}^{k} \times \mathbb{H}^{n-k}=\left\{\mathbf{u} \in \mathbb{R}_{1}^{n+1} \mid-u_{1}^{2}+\sum_{i=k+2}^{n+1} u_{i}^{2}=\frac{1}{r}, r<0\right\}$ with $\mathbf{A}= \pm\left(\mathbf{0}_{k} \oplus \sqrt{-r} \mathcal{I}_{n-k}\right)$, 
(4) $\mathbb{R}_{1}^{n}=\left\{\mathbf{u} \in \mathbb{R}_{1}^{n+1} \mid u_{n+1}=0\right\}$ with $\mathbf{A}=\mathbf{0}_{n}$,

(5) $\mathbb{S}_{1}^{n}(r)=\left\{\mathbf{u} \in \mathbb{R}_{1}^{n+1} \mid-u_{1}^{2}+\sum_{i=2}^{n+1} u_{i}^{2}=\frac{1}{r}, r>0\right\}$ with $\mathbf{A}= \pm \sqrt{r} \mathcal{I}_{n}$,

(6) $\mathbb{R}^{k} \times \mathbb{S}_{1}^{n-k}(r)=\left\{\mathbf{u} \in \mathbb{R}_{1}^{n+1} \mid-u_{1}^{2}+\sum_{i=k+2}^{n+1} u_{i}^{2}=\frac{1}{r}, r>0\right\}$ with $\mathbf{A}= \pm\left(\mathbf{0}_{k} \oplus \sqrt{r} \mathcal{I}_{n-k}\right)$.

While the first three of above hypersurfaces are spacelike, the others are Lorentzian. According to this classification, we consider the Pythagorean isoparametric hypersurfaces.

Theorem 5. Let $M$ be a complete isoparametric hypersurface with at most two mutually distinct principal curvatures in $\mathbb{R}_{1}^{n+1}$ having diagonalizable shape operator. If $M$ is Pythagorean, then it is isometric to $\mathbb{H}^{n}(-\varphi)$ or $\mathbb{S}_{1}^{n}(\varphi)$.

Proof. By the hypothesis and Remark 1, M may be one of the above items (2), (3), (5) and (6). The observation of these items will be separate.

Cases (2) and (5). $\mathbf{A}= \pm \sqrt{-r} \mathcal{I}_{n}, r<0$. Then (1) gives

$$
\left(r^{2}+r-1\right) \mathbf{I}^{2}=\mathbf{0}_{n}
$$

which implies $r=-\varphi$ since $\mathbf{I}$ is invertible. Then $M$ is isometric to $\mathbb{H}^{n}(-\varphi)$. A similar calculation gives $r=\varphi$ for the case $\mathbf{A}= \pm \sqrt{r} \mathcal{I}_{n}, r>0$ and in such a case $M$ is isometric to $\mathbb{S}_{1}^{n}(\varphi)$. Then the result follows.

Cases (3) and (6). $\mathbf{A}= \pm\left(\mathbf{0}_{k} \oplus \sqrt{-r} \mathcal{I}_{n-k}\right), r<0$. We have

$$
\mathbf{A}^{2}=\mathbf{0}_{k} \oplus(-r) \mathcal{I}_{n-k}
$$

and

$$
\mathbf{I} \cdot \mathbf{A}=\mathbf{0}_{n-k} \oplus( \pm) \sqrt{-r} \mathbf{I}_{k} .
$$

By these two equations, (1) implies:

$$
\mathbf{I}^{2}=\mathbf{0}_{n-k} \oplus\left(r+r^{2}\right) \mathbf{I}_{k}^{2} .
$$

After taking determinant in both hand sides, we obtain the contradiction $\operatorname{det} \mathbf{I}=0$. By using a similar argument, we obtain a contradiction for the case $\mathbf{A}= \pm\left(\mathbf{0}_{k} \oplus \sqrt{r} \mathcal{I}_{n-k}\right)$, too.

Notice that the sectional curvatures of the Pythagorean hypersurfaces $\mathbb{H}^{n}(-\varphi)$ and $\mathbb{S}_{1}^{n}(\varphi)$ are $K= \pm \varphi$ and the scalar curvatures $\tau= \pm\left(\begin{array}{c}n \\ 2\end{array}\right) \varphi$.

\subsection{Hypersurfaces of the Anti-de Sitter Space}

An isoparametric hypersurface $M$ with at most two mutually distinct principal curvatures in $\mathbb{H}_{1}^{n+1}(-1):=\mathbb{H}_{1}^{n+1}$ is given by $([20])$ :

(1) $\mathbb{H}^{n}(r)=\left\{\mathbf{u} \in \mathbb{H}_{1}^{n+1} \subset \mathbb{R}_{2}^{n+2} \mid u_{1}=\sqrt{\frac{1}{r}+1}, r \leq-1\right\}$ with $\mathbf{A}= \pm \sqrt{-1-r} \mathcal{I}_{n}$ ，

(2) $\mathbb{H}^{k}\left(r_{1}\right) \times \mathbb{H}^{n-k}\left(r_{2}\right)=\left\{\mathbf{u} \in \mathbb{H}_{1}^{n+1} \subset \mathbb{R}_{2}^{n+2} \mid-u_{1}^{2}+\sum_{i=3}^{k+2} u_{i}^{2}=\frac{1}{r_{1}},-u_{2}^{2}+\sum_{i=k+3}^{n+2} u_{i}^{2}=\right.$ $\left.\frac{1}{r_{2}}\right\},\left(\frac{1}{r_{1}}+\frac{1}{r_{2}}=-1, r_{1}, r_{2}<0\right)$ with $\mathbf{A}= \pm\left(\sqrt{-1-r_{1}} \mathcal{I}_{k} \oplus\left(-\sqrt{-1-r_{2}}\right) \mathcal{I}_{n-k}\right)$.

(3) $\mathbb{R}_{1}^{n}=\left\{\mathbf{u} \in \mathbb{H}_{1}^{n+1} \subset \mathbb{R}_{2}^{n+2} \mid u_{1}=u_{n+2}+s_{0}, s_{0}>0\right\}$ with $\mathbf{A}= \pm \mathcal{I}_{n}$ ，

(4) $\mathbb{S}_{1}^{n}(r)=\left\{\mathbf{u} \in \mathbb{H}_{1}^{n+1} \subset \mathbb{R}_{2}^{n+2} \mid u_{1}=\sqrt{\frac{r+1}{r}}, r>0\right\}$ with $\mathbf{A}= \pm \sqrt{r+1} \mathcal{I}_{n}$,

(5) $\mathbb{H}_{1}^{n}(r)=\left\{\mathbf{u} \in \mathbb{H}_{1}^{n+1} \subset \mathbb{R}_{2}^{n+2} \mid u_{n+2}=\sqrt{-\frac{r+1}{r}},-1 \leq r<0\right\}, \mathbf{A}= \pm \sqrt{r+1} \mathcal{I}_{n}$,

(6) $\mathbb{S}_{1}^{r}\left(r_{1}\right) \times \mathbb{H}^{n-r}\left(r_{2}\right)=\left\{\mathbf{u} \in \mathbb{H}_{1}^{n+1} \subset \mathbb{R}_{2}^{n+2} \mid-u_{1}^{2}+\sum_{i=3}^{k+2} u_{i}^{2}=\frac{1}{r_{1}},-u_{2}^{2}+\sum_{i=k+3}^{n+2} u_{i}^{2}=\frac{1}{r_{2}}\right\}$, $\left(\frac{1}{r_{1}}+\frac{1}{r_{2}}=1, r_{1}>0, r_{2}<0\right), \mathbf{A}= \pm\left(\sqrt{1+r_{1}} \mathcal{I}_{k} \oplus \sqrt{1+r_{2}} \mathcal{I}_{n-k}\right)$. 
Here the non-degenerate metric of $\mathbb{R}_{2}^{n+2}$ is of the form $-d u_{1}^{2}-d u_{2}^{2}+\sum_{i=3}^{n+2} d u_{i}^{2}$. While the first two of above hypersurfaces are spacelike, the others are Lorentzian. Then we have the next result:

Theorem 6. Let $M$ be a complete isoparametric hypersurface with at most two mutually distinct principal curvatures in $\mathbb{H}_{1}^{n+1}$ having diagonalizable shape operator. Then $M$ is Pythagorean if and only if it is isometric to either $\mathbb{H}^{n}(-(1+\varphi))$ or $\mathbb{S}_{1}^{n}(\Phi)$ or $\mathbb{S}_{1}^{k}(\Phi) \times \mathbb{H}^{n-k}(-\varphi)$.

Proof. We may neglect the above item (3) according to Remark 1 . We observe the other items, separately.

Case (1). $\mathbf{A}= \pm \sqrt{-r-1} \mathcal{I}_{n}, r \leq-1$. Then (1) gives:

$$
\left(r^{2}+3 r+1\right) \mathbf{I}^{2}=\mathbf{0}_{n}
$$

It follows that $r^{2}+3 r+1=0$ since $\mathbf{I}$ is invertible. The only permissible root is $r=-(1+\varphi)$ and hence $M$ is isometric to $H^{n}(-(1+\varphi))$.

Case (2). $\mathbf{A}= \pm\left(\sqrt{-1-r_{1}} \mathcal{I}_{k} \oplus\left(-\sqrt{-1-r_{2}}\right) \mathcal{I}_{n-k}\right), \frac{1}{r_{1}}+\frac{1}{r_{2}}=-1, r_{1}, r_{2}<0$. We write

$$
\mathbf{A}^{2}=-\left(\left(1+r_{1}\right) \mathcal{I}_{k} \oplus\left(1+r_{2}\right) \mathcal{I}_{n-k}\right)
$$

and

$$
\mathbf{A}^{4}=\left(1+r_{1}\right)^{2} \mathcal{I}_{k} \oplus\left(1+r_{2}\right)^{2} \mathcal{I}_{n-k} .
$$

After choosing an orthogonal frame of $M$ such that $\mathbf{I}$ is diagonal and $\mathbf{A}$ as in our case, we conclude from (1) that:

$$
\mathbf{I}^{2}\left(-\mathcal{I}_{n}-\mathbf{A}^{2}+\mathbf{A}^{4}\right)=\mathbf{0}_{n}
$$

Then, it follows:

$$
\begin{aligned}
& r_{1}^{2}+3 r_{1}+1=0 \\
& r_{2}^{2}+3 r_{2}+1=0
\end{aligned}
$$

which have the roots $r_{1}, r_{2} \in\{-1-\varphi, \phi-2\}$. Since $\frac{1}{r_{1}}+\frac{1}{r_{2}}=-1$, we find a contradiction.

Cases (4) and (5). $\mathbf{A}= \pm \sqrt{r+1} \mathcal{I}_{n}, r>0$. Then (1) gives:

$$
\left(r^{2}+r-1\right) \mathbf{I}^{2}=\mathbf{0}_{n}
$$

where $r=\Phi$ due to $\operatorname{det} \mathbf{I} \neq 0$. Then $M$ is isometric to $S_{1}^{n}(\Phi)$. For the case $\mathbf{A}= \pm \sqrt{r+1} \mathcal{I}_{n}$, $-1 \leq r<0$, a contradiction may be obtained by a similar calculation.

Case (6). $\mathbf{A}= \pm\left(\sqrt{1+r_{1}} \mathcal{I}_{k} \oplus\left(\sqrt{1+r_{2}}\right) \mathcal{I}_{n-k}\right), \frac{1}{r_{1}}+\frac{1}{r_{2}}=1, r_{1}>0, r_{2}<0$.

We choose an orthonormal frame of $M$ such that $\mathbf{I}$ is diagonal. We then conclude from (1) that:

$$
\mathbf{I}^{2}\left(-\mathcal{I}_{n}-\mathbf{A}^{2}+\mathbf{A}^{4}\right)=\mathbf{0}_{n}
$$

We have

$$
\mathbf{A}^{2}=\left(1+r_{1}\right) \mathcal{I}_{k} \oplus\left(1+r_{2}\right) \mathcal{I}_{n-k}
$$

and

$$
\mathbf{A}^{4}=\left(1+r_{1}\right)^{2} \mathcal{I}_{k} \oplus\left(1+r_{2}\right)^{2} \mathcal{I}_{n-k} .
$$

By replacing the values of $\mathbf{A}^{2}$ and $\mathbf{A}^{4}$, we obtain:

$$
\begin{aligned}
& r_{1}^{2}+r_{1}-1=0 \\
& r_{2}^{2}+r_{2}-1=0 .
\end{aligned}
$$


The solutions are $r_{1}=\Phi$ and $r_{2}=-\varphi$. Then $M$ is isometric to $\mathbb{S}_{1}^{k}(\Phi) \times \mathbb{H}^{n-k}(-\varphi)$, completing the proof.

Notice that the sectional curvatures of the Pythagorean hypersurfaces $\mathbb{H}^{n}(-(1+\varphi))$ and $\mathbb{S}_{1}^{n}(\Phi)$ are $K=-1 \pm \varphi$ and the scalar curvatures are $\tau=\left(\begin{array}{c}n \\ 2\end{array}\right)(-1 \pm \varphi)$.

\subsection{Hypersurfaces of the de Sitter Space}

An isoparametric hypersurface $M$ with at most two mutually distinct principal curvatures in $\mathbb{S}_{1}^{n+1}(1):=\mathbb{S}_{1}^{n+1}$ is given by ([20]):

(1) $\mathbb{R}^{n}=\left\{\mathbf{u} \in \mathbb{S}_{1}^{n+1} \subset \mathbb{R}_{1}^{n+2} \mid u_{1}=u_{n+2}+s_{0}, s_{0}>0\right\}, \mathbf{A}= \pm \mathcal{I}_{n}$ ，

(2) $\mathbb{S}^{n}(r)=\left\{\mathbf{u} \in \mathbb{S}_{1}^{n+1} \subset \mathbb{R}_{1}^{n+2} \mid u_{1}=\sqrt{\frac{1}{r}-1}, 0<r \leq 1\right\}, \mathbf{A}= \pm \sqrt{1-r} \mathcal{I}_{n}$,

(3) $\mathbb{H}^{n}(r)=\left\{\mathbf{u} \in \mathbb{S}_{1}^{n+1} \subset \mathbb{R}_{1}^{n+2} \mid u_{n+2}=\sqrt{1-\frac{1}{r}}, r<0\right\}, \mathbf{A}= \pm \sqrt{1-r} \mathcal{I}_{n}$,

(4) $\mathbb{S}^{k}\left(r_{1}\right) \times \mathbb{H}^{n-k}\left(r_{2}\right)=\left\{\mathbf{u} \in \mathbb{S}_{1}^{n+1} \subset \mathbb{R}_{1}^{n+2} \mid \sum_{i=1}^{k+2} u_{i}^{2}=\frac{1}{r_{1}},-u_{1}^{2}+\sum_{i=k+3}^{n+2} u_{i}^{2}=\frac{1}{r_{2}}\right\}$, $\left(\frac{1}{r_{1}}+\frac{1}{r_{2}}=1, r_{1}>0, r_{2}<0\right), \mathbf{A}= \pm\left(\sqrt{1-r_{1}} \mathcal{I}_{k} \oplus \sqrt{1-r_{2}} \mathcal{I}_{n-k}\right)$,

(5) $\mathbb{S}_{1}^{n}(r)=\left\{\mathbf{u} \in \mathbb{S}_{1}^{n+1} \subset \mathbb{R}_{1}^{n+2} \mid u_{n+2}=\sqrt{1-\frac{1}{r}}, r \geq 1\right\}, \mathbf{A}= \pm \sqrt{r-1} \mathcal{I}_{n}$,

(6) $\mathbb{S}^{k}\left(r_{1}\right) \times \mathbb{S}_{1}^{n-k}\left(r_{2}\right)=\left\{\mathbf{u} \in \mathbb{S}_{1}^{n+1} \subset \mathbb{R}_{1}^{n+2} \mid \sum_{i=2}^{k+2} u_{i}^{2}=\frac{1}{r_{1}},-u_{1}^{2}+\sum_{i=k+3}^{n+2} u_{i}^{2}=\frac{1}{r_{2}}\right\}$, $\left(\frac{1}{r_{1}}+\frac{1}{r_{2}}=1, r_{1}>0, r_{2}>0\right), \mathbf{A}= \pm\left(\sqrt{r_{1}-1} \mathcal{I}_{k} \oplus\left(-\sqrt{r_{2}-1} \mathcal{I}_{n-k}\right)\right)$.

Here the non-degenerate metric of $\mathbb{R}_{1}^{n+2}$ is of the form $-d u_{1}^{2}+\sum_{i=2}^{n+2} d u_{i}^{2}$. While the last two of above hypersurfaces are Lorentzian, the others are spacelike. We can state the following theorem:

Theorem 7. Let $M$ be a complete isoparametric hypersurface with at most two mutually distinct principal curvatures in $\mathbb{S}_{1}^{n+1}$ having diagonalizable shape operator. If $M$ is Pythagorean, then it is isometric to $\mathbb{H}^{n}(-\Phi)$ or $\mathbb{S}_{1}^{n}(1+\varphi)$.

Proof. The above item (1) may be neglected according to Remark 1 . We observe the other items, separately.

Cases (2) and (3). $\mathbf{A}= \pm \sqrt{-r+1} \mathcal{I}_{n}, 0<r \leq 1$. Then (1) gives

$$
\left(r^{2}-r-1\right) \mathbf{I}^{2}=\mathbf{0}_{n}
$$

It follows that $r^{2}-r-1=0$ since $\mathbf{I}$ is invertible. However it has no root in the interval $(0,1]$. By a similar calculation, in the case $r<0$ we may derive that $M$ is isometric to $H^{n}(-\Phi)$.

Case (4). $\mathbf{A}= \pm\left(\sqrt{1-r_{1}} \mathcal{I}_{k} \oplus\left(\sqrt{1-r_{2}}\right) \mathcal{I}_{n-k}\right), \frac{1}{r_{1}}+\frac{1}{r_{2}}=1, r_{1}>0, r_{2}<0$.

We have

$$
\mathbf{A}^{2}=\left(1-r_{1}\right) \mathcal{I}_{k} \oplus\left(1-r_{2}\right) \mathcal{I}_{n-k}
$$

and

$$
\mathbf{A}^{4}=\left(1-r_{1}\right)^{2} \mathcal{I}_{k} \oplus\left(1-r_{2}\right)^{2} \mathcal{I}_{n-k} .
$$

After choosing an orthonormal frame of $M$ such that $\mathbf{I}$ is diagonal, we conclude from (1) that:

$$
\mathbf{I}^{2}\left(-\mathcal{I}_{n}-\mathbf{A}^{2}+\mathbf{A}^{4}\right)=\mathbf{0}_{n} .
$$

Then, it follows:

$$
\begin{aligned}
& r_{1}^{2}-r_{1}-1=0, \\
& r_{2}^{2}-r_{2}-1=0,
\end{aligned}
$$

which have the roots $r_{1}=\varphi$ and $r_{2}=-\Phi$. This however contradicts $\frac{1}{r_{1}}+\frac{1}{r_{2}}=1$. 
Case (5). $\mathbf{A}= \pm \sqrt{r-1}, r \geq 1$. Then (1) gives:

$$
\left(r^{2}-3 r+1\right) \mathbf{I}^{2}=\mathbf{0}_{n}
$$

where $r=1+\varphi$, due to $\operatorname{det} \mathbf{I} \neq 0$. Then $M$ is isometric to $S_{1}^{n}(1+\varphi)$.

Case (6). $\mathbf{A}= \pm\left(\sqrt{r_{1}-1} \mathcal{I}_{k} \oplus\left(-\sqrt{r_{2}-1}\right) \mathcal{I}_{n-k}\right), \frac{1}{r_{1}}+\frac{1}{r_{2}}=1, r_{1}, r_{2}>0$.

We choose an orthonormal frame of $M$ such that $\mathbf{I}$ is diagonal. We then conclude from (1) that:

$$
\mathbf{I}^{2}\left(-\mathcal{I}_{n}-\mathbf{A}^{2}+\mathbf{A}^{4}\right)=\mathbf{0}_{n}
$$

We have

$$
\mathbf{A}^{2}=\left(r_{1}-1\right) \mathcal{I}_{k} \oplus\left(r_{2}-1\right) \mathcal{I}_{n-k}
$$

and

$$
\mathbf{A}^{4}=\left(r_{1}-1\right)^{2} \mathcal{I}_{k} \oplus\left(r_{2}-1\right)^{2} \mathcal{I}_{n-k} .
$$

By replacing the values of $\mathbf{A}^{2}$ and $\mathbf{A}^{4}$, we obtain:

$$
\begin{aligned}
& r_{1}^{2}-3 r_{1}+1=0 \\
& r_{2}^{2}-3 r_{2}+1=0 .
\end{aligned}
$$

The solutions are $r_{1}=1+\varphi, r_{1}=1-\Phi$ and $r_{2}=1+\varphi, r_{2}=1-\Phi$. However this is not possible since $\frac{1}{r_{1}}+\frac{1}{r_{2}}=1$; this completes the proof.

Notice that the sectional curvatures of the Pythagorean hypersurfaces $\mathbb{H}^{n}(-\Phi)$ and $\mathbb{S}_{1}^{n}(1+\varphi)$ are $K=1 \pm \varphi$ and the scalar curvatures are $\tau=\left(\begin{array}{l}n \\ 2\end{array}\right)(1 \pm \varphi)$.

Summarizing the obtained results in Lorentzian ambient space, more precisely Theorems 5-7, we give the following classification result:

Theorem 8 (Classification of Pythagorean isoparametric hypersurfaces of a Lorentzian space form). Let $M$ be a complete isoparametric hypersurface with at most two mutually distinct principal curvatures in a Lorentzian space form $\mathbb{M}_{1}^{n+1}(c), c \in\{-1,0,1\}$ having diagonalizable shape operator. If $M$ is Pythagorean, then it is either totally umbilical with $K= \pm \varphi+c$ or isometric to $\mathbb{S}_{1}^{k}(\Phi) \times \mathbb{H}^{n-k}(-\varphi)$.

We point out that $\mathbb{S}_{1}^{k}(\Phi) \times \mathbb{H}^{n-k}(-\varphi)$ is the only isoparametric Pythagorean hypersurface in our investigation which is not totally umbilical.

Author Contributions: All authors contributed equally to this research. The research was carried out by all the authors, and the manuscript was subsequently prepared together. All authors have read and agreed to the published version of the manuscript.

Funding: This research received no external funding.

Conflicts of Interest: The authors declare no conflict of interest.

\section{References}

1. Takloo-Bighash, R. A Pythagorean Introduction to Number Theory. Right Triangles, Sums of Squares, and Arithmetic; Undergraduate Texts in Mathematics; Springer: Cham, Switzerland, 2018.

2. Palmer, L.; Ahuja, M.; Tikoo, M. Finding Pythagorean triple preserving matrices. Missouri J. Math. Sci. 1998, 10, 99-105. [CrossRef]

3. Crasmareanu, M. A new method to obtain Pythagorean triple preserving matrices. Missouri J. Math. Sci. 2002, 14, 149-158. [CrossRef]

4. Palmer, L.; Ahuja, M.; Tikoo, M. Constructing Pythagorean triple preserving matrices. Missouri J. Math. Sci. 1998, 10, 159-168. [CrossRef]

5. Arnold, M.; Eydelzon, A. On matrix Pythagorean triples. Amer. Math. Mon. 2019, 126, 158-160. [CrossRef]

6. Aydin, M.E.; Mihai, A. A note on surfaces in space forms with Pythagorean fundamental forms. Mathematics 2020, 8, 444.

7. Gray, A. Modern Differential Geometry of Curves and Surfaces with Mathematica, 2nd ed.; CRC Press: Boca Raton, FL, USA, 1998.

8. Dunlap, R.A. The Golden Ratio and Fibonacci Numbers; World Scientific Publishing Co. Inc.: River Edge, NJ, USA, 1997.

9. Livio, M. The Golden Ratio. The Story of Phi, the World's Most Astonishing Number; Broadway Books: New York, NY, USA, 2002. 
10. Chen, B.-Y. Differential Geometry of Warped Product Manifolds and Submanifolds; World Scientific Publishing Co. Pte. Ltd.: Hackensack, NJ, USA, 2017.

11. Cecil, T.E. Isoparametric and Dupin hypersurfaces. SIGMA Symmetry Integr. Geom. Methods Appl. 2008, 4, 62. [CrossRef]

12. Özgür, C.; Özgür, N.Y. Classification of metallic shaped hypersurfaces in real space forms. Turk. J. Math. 2015, 39, 784-794. [CrossRef]

13. Özgür, C.; Özgür, N.Y. Metallic shaped hypersurfaces in Lorentzian space forms. Rev. Un. Mat. Argent. 2017, 58, 215-226.

14. Crasmareanu, M.; Hretcanu, C.-E.; Munteanu, M.-I. Golden- and product-shaped hypersurfaces in real space forms. Int. J. Geom. Methods Mod. Phys. 2013, 10, 1320006. [CrossRef]

15. Chen, B.-Y. Geometry of Submanifolds. In Pure and Applied Mathematics 22; Marcel Dekker, Inc.: New York, NY, USA, 1973.

16. Kuhnel, W. Differential Geometry: Curves-Surfaces-Manifolds, 2nd ed.; AMS: Providence, RI, USA, 2002.

17. Ryan, P.J. Hypersurfaces with parallel Ricci tensor. Osaka Math. J. 1971, 8, 251-259.

18. Ryan, P.J. Homogeneity and some curvature conditions for hypersurfaces. Tohoku Math. J. 1969, 21, 363-388. [CrossRef]

19. Magid, M.A. Lorentzian isoparametric hypersurfaces. Pacific J. Math. 1985, 118, 165-197. [CrossRef]

20. Abe, N.; Koike, N.; Yamaguchi, S. Congruence theorems for proper semi-Riemannian hypersurfaces in a real space form. Yokohama Math. J. 1987, 35, 123-136. 